\title{
Estudio de la Implantación de diversas Competencias Transversales en Asignaturas de diferentes Titulaciones de Ingeniería de la UPV
}

Silvia Alvarez Blanco ${ }^{a}$, Ignacio Bosch Roig ${ }^{b}$, Cristina Jordan Lluch ${ }^{c}$, Jaime Lloret ${ }^{b}$, José Antonio Mendoza ${ }^{a}$, Lucia Romero Perez ${ }^{d}$, Esther Sanabria-Codesal ${ }^{\mathrm{c}}$ y Maria Cinta Vincent-Vela ${ }^{a}$

a Departamento de Ingeniería Química y Nuclear (sialvare@iqn.upv.es, mavinve@iqn.upv.es, jamendoz@iqn.upv.es), ${ }^{\mathrm{b}}$ Departamento de Comunicaciones (igbosroi@dcom.upv.es,

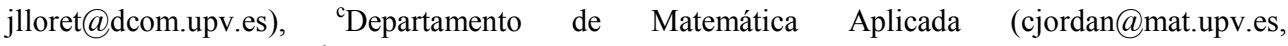
esanabri@mat.upv.es)y ${ }^{\mathrm{d}}$ Departamento de Ingeniería Electrónica (lurope@eln.upv.es)

\begin{abstract}
This article shows the tracking of the transversal competences applied to different subjects in different engineering degrees of the Polytechnic University of Valencia (UPV), allowing us to obtain a broad view on the transversal competences. We focus on the comparison of the materials developed in each subject for the design and proposed activities of teaching learning as well as in the design of specific evaluation instruments, obtaining evidence, etc. Finally, the evaluation strategies carried out as well as the obtained results are shown. The subjects analyzed in this paper are related because the belong to Engineering taught at UPV, by members of the teaching innovation group EITACURTE, which make periodic meetings in order to exchange ideas about their teaching experiences.
\end{abstract}

Keywords: Transversal skills, teaching-learning monitoring, Grade.

\begin{abstract}
Resumen
En este artículo se realiza un seguimiento del trabajo realizado en competencias transversales en diferentes asignaturas de distintas titulaciones de ingeniería de la Universitat Politècnica de València (UPV), permitiéndonos obtener una visión amplia sobre éstas. Nos centramos en la comparación de los materiales desarrollados en cada asignatura para el diseño y propuesta de actividades de enseñanza - aprendizaje, así como en el diseño de instrumentos de evaluación y la recopilación de evidencias. Finalmente se muestran las estrategias de evaluación llevadas a cabo, así como los resultados obtenidos. Las asignaturas analizadas en este artículo son impartidas por los profesores pertenecientes al grupo de innovación docente EITACURTE, los cuales realizan reuniones periódicas para intercambiar ideas sobre sus experiencias docentes.
\end{abstract}

Palabras clave: Competencias transversales, Seguimiento enseñanzaaprendizaje, Grado. 


\section{Introducción}

Las competencias transversales, siguiendo lo propuesto por Gonzalez y Wagenaar, 2003, son destrezas relacionadas con el desarrollo personal, que no dependen de un ámbito temático o disciplinario específico sino que aparecen en todos los dominios de la actuación profesional y académica. La Universidad Politécnica de Valencia (UPV), de acuerdo con el plan de incorporación progresiva de las competencias transversales a sus títulos, ha decidido que en las guías docentes se incluyan las estas como competencias a elegir por los docentes que han sido seleccionados como puntos de control por las diferentes Estructuras Responsables de Títulos (ERT) (Sanabria-Codesal et al. 2014). La UPV considera que las competencias transversales que deben tener sus egresados de grado y máster son las que figuran en la siguiente tabla:

Tabla 1. Competencias Transversales de la UPV

\begin{tabular}{|l|l|}
\hline Acrónimo & Título \\
\hline CT01 & Comprensión e integración \\
\hline CT02 & Aplicación y pensamiento práctico \\
\hline CT03 & Análisis y resolución de problemas \\
\hline CT04 & Innovación, creatividad y emprendimiento \\
\hline CT05 & Diseño y proyecto \\
\hline CT06 & Trabajo en equipo y liderazgo \\
\hline CT07 & Responsabilidad ética, medioambiental y profesional \\
\hline CT08 & Comunicación efectiva \\
\hline CT09 & Pensamiento critico \\
\hline CT10 & Conocimiento de problemas contemporáneos \\
\hline CT11 & Aprendizaje permanente \\
\hline CT12 & Planificación y gestión de tiempo \\
\hline CT13 & Instrumental específica \\
\hline
\end{tabular}

Esto ha provocado que en la actualidad se esté trabajando intensamente en la adecuación de las guías docentes de los títulos UPV a este requerimiento, lo que ha posibilitado la creación de diversos grupos de trabajo para coordinar cada una de las competencias y buscar aspectos comunes, métodos de evaluación, resultados de aprendizaje, etc. extrapolables a cualquier materia impartida en sus centros.

Para llevar a cabo una correcta implantación, es necesario un seguimiento y revisión detallada de las diferentes acciones para enseñar y evaluar adecuadamente las competencias

(c) EY-NC-ND 2016, Universitat Politècnica de València 
transversales (Sendra et al., 2009). Además, se debe comprobar si éstas se están evaluando en diversas asignaturas dentro de cada grado para asegurar que los alumnos realmente están obteniendo el nivel de dominio requerido en estos conocimientos colaterales tan demandados por la sociedad en la actualidad.

El presente artículo muestra estrategias de trabajo y evaluación de diferentes competencias transversales, llevadas a cabo en diferentes asignaturas de grado y máster, así como los resultados obtenidos en dichas evaluaciones.

El resto del artículo está estructurado como sigue: en la sección 2 se muestran los objetivos y en la sección 3 se hace una revisión de trabajos relacionados. Las secciones 4 y 5 presentan las asignaturas donde se han trabajado las diferentes competencias transversales, así como los resultados obtenidos. Finalmente, en la sección 6 se exponen las conclusiones y los trabajos futuros.

\section{Objetivos}

Los objetivos específicos que se pretenden trabajar aquí son los siguientes:

- Analizar y comprender las competencias transversales UPV aplicadas en las asignaturas de diversas titulaciones UPV.

- Comprobar que las competencias transversales aplicadas en estas asignaturas sean realistas y se ajusten a los objetivos de los profesores en cada materia.

- Realizar una comparación de los materiales desarrollados en cada asignatura para trabajar las diferentes competencias transversales con actividades de enseñanza - aprendizaje.

- Valorar el diseño de los instrumentos de evaluación utilizados que permiten la obtención de resultados específicos en las competencias transversales de cada asignatura.

\section{Trabajos Relacionados}

La importancia del desarrollo de las competencias transversales ha sido establecida con la aparición de la acreditación ABET para ingenierías (ABET, 1997) (ABET, 2008), aunque hasta el momento no se ha establecido un método detallado para la evaluación de éstas (Kyoung Ro, 2015). Las diferentes formas de evaluar dichas competencias deberían ser validadas por un organismo regulador, puesto que se han observado algunas dificultades, entre otras: carencia de una definición comprensible y adecuada de las mismas, limitaciones metodológicas para su evaluación, inexistencia de estudios de validez del proceso de evaluación de las competencias (Kilgore, 2007).

Diversos trabajos muestran que las diferentes metodologías educativas usadas por los profesores afectan a la percepción del desarrollo de las competencias transversales por parte de los alumnos (Bjorklund, 2004). Las últimas reformas en materia de educación superior van encaminadas a ayudar a los estudiantes a desarrollar sus habilidades (Redish, 2008),

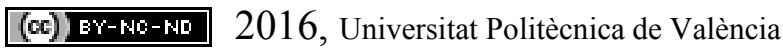

Congreso In-Red (2016) 
siendo los 3 pilares del curriculum de un Ingeniero (Jamieson, 2007), mostrados en la Figura 1, una guía para fijar las competencias más adecuadas en las nuevas titulaciones.
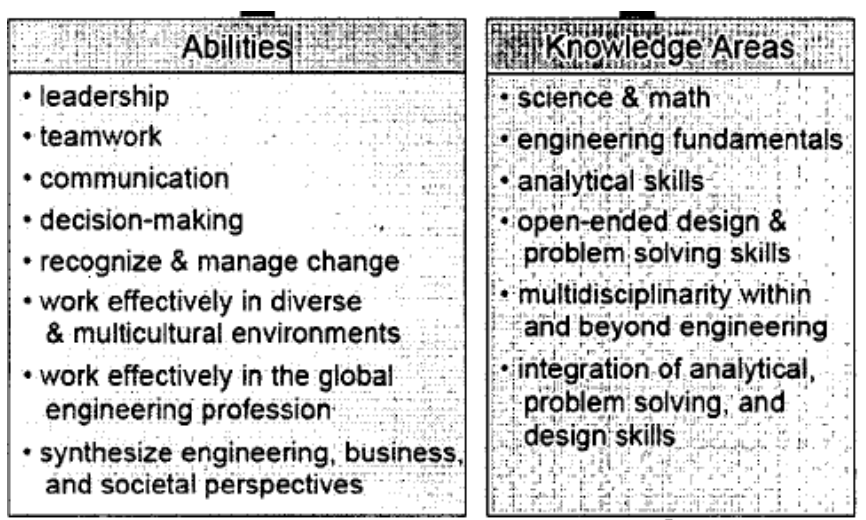

\begin{tabular}{|l|}
\hline Qualities \\
\hline - innovative \\
- strong work ethic \\
global, social, intellectual, \\
and technological context \\
- adaptable in a changing \\
environment \\
entrepreneurial and \\
intrapreneurial \\
curious and persistent \\
continuous learners \\
\end{tabular}

Fig 1. Pilares del Curriculum del Ingeniero

\section{Competencias transversales en diversas asignaturas}

En esta sección se presentan diversas asignaturas de las titulaciones de la UPV donde se han trabajado competencias transversales y se explica, en cada una de ellas, la metodología utilizada, así como los métodos para evaluarlas.

La experiencia se ha realizado en diferentes asignaturas, niveles competenciales y grados de la, como se muestra en la Tabla 2.

Tabla 2. Asignaturas UPV en las que se han trabajado competencias transversales

\begin{tabular}{|c|c|c|c|c|}
\hline Asignatura & Grado & Competencia & $\begin{array}{l}\text { Curso / Nivel } \\
\text { competencial }\end{array}$ & Cuatrimestre \\
\hline $\begin{array}{l}\text { Matemática } \\
\text { Discreta }\end{array}$ & $\begin{array}{c}\text { Grado en } \\
\text { Informática }\end{array}$ & CT10 & $\begin{array}{c}1^{\circ} \text { Grado / } \\
\text { Nivel I }\end{array}$ & A \\
\hline $\begin{array}{l}\text { Señales y } \\
\text { Sistemas }\end{array}$ & $\begin{array}{c}\text { Grado en } \\
\text { Ingeniería de } \\
\text { Tecnologías y } \\
\text { Servicios de } \\
\text { Telecomunicación }\end{array}$ & СТ02 & $\begin{array}{c}2^{\circ} \text { Grado / } \\
\text { Nivel I }\end{array}$ & A \\
\hline $\begin{array}{c}\text { Matemáticas } \\
\text { II }\end{array}$ & $\begin{array}{l}\text { Grado en } \\
\text { Ingeniería } \\
\text { Mecánica }\end{array}$ & CT12 & $\begin{array}{c}2^{\circ} \text { Grado / } \\
\text { Nivel I }\end{array}$ & A \\
\hline $\begin{array}{c}\text { Sistemas } \\
\text { Electrónicos }\end{array}$ & $\begin{array}{c}\text { Grado en } \\
\text { Ingeniería en } \\
\text { Tecnologías } \\
\text { Industriales }\end{array}$ & СТ09 & $\begin{array}{c}3^{\circ} \text { Grado / } \\
\text { Nivel II }\end{array}$ & A \\
\hline $\begin{array}{c}\text { Tratamiento } \\
\text { biológico de } \\
\text { aguas } \\
\text { residuales }\end{array}$ & $\begin{array}{l}\text { Máster en } \\
\text { Ingeniería } \\
\text { Química }\end{array}$ & CT10 & $\begin{array}{c}2^{\circ} \text { Máster / } \\
\text { Nivel III }\end{array}$ & A \\
\hline
\end{tabular}

(c) EY-NC-ND 2016, Universitat Politècnica de València 


\subsection{Competencia CT10: Conocimiento de problemas contemporáneos (Matemática Discreta)}

La competencia Conocimiento de problemas contemporáneos se ha trabajado y evaluado en la asignatura Matemática Discreta (MAD). Esta asignatura corresponde al primer cuatrimestre del primer curso del grado en Ingeniería Informática que se imparte en la Escuela Técnica Superior de Ingeniería Informática (ETSINF) de la UPV y tiene 10 grupos que se desdoblan en 2 subgrupos de prácticas.

MAD tiene asignados 4,5 créditos en aula y 1,5 en laboratorio informático. El temario impartido en aula contiene los temas: Lógica, Conjuntos, Relaciones binarias, Divisibilidad, Álgebras de Boole y Combinatoria, dedicando las sesiones de laboratorio al estudio introductorio de la Teoría de Grafos. Esta asignatura es punto de control, es decir, se evalúa a los alumnos que la cursan, en dos competencias transversales, la CT03: Análisis y resolución de problemas y la CT10: Conocimiento de problemas contemporáneos, objeto de estudio en este trabajo.

La Teoría de Grafos, impartida en el laboratorio de esta asignatura, está experimentado un gran auge en la actualidad debido a su gran aplicabilidad en todas las ciencias, por lo que constituye una materia especialmente adecuada para la competencia transversal que nos ocupa. Es de señalar que el enfoque que se le da a esta parte de la asignatura es eminentemente práctico, algorítmico, encaminado a la resolución de muy diversos problemas de la vida real, obviamente escalados al nivel de conocimiento de nuestros alumnos. Por ello, desde un primer momento, al iniciar cada tema se plantean problemas reales, introduciendo y desarrollando la teoría como elemento necesario para su resolución.

La evaluación de esta parte de la materia se lleva a cabo mediante examen. En la primera parte de este se plantean cuestiones más teóricas, básicas para el correcto conocimiento y manejo de esta teoría y del conocimiento de los algoritmos estudiados. En la segunda parte se propone un problema real, con diferentes apartados, cuya resolución pasa por una primera etapa de modelización, utilizando la teoría de grafos, siendo necesario analizar a continuación el algoritmo que es conveniente aplicar y resolver con ayuda del software para terminar finalmente interpretando los resultados proporcionados.

En el curso académico 2015-16, la competencia se ha evaluado de manera proporcional a la nota obtenida en el examen de prácticas, estando previsto que el curso próximo que su calificación se obtenga a partir únicamente de la nota obtenida por el alumno en la parte de modelización de dicho examen.4.2 Competencia CT02: Aplicación y pensamiento práctico (Señales y Sistemas)

La asignatura Señales y Sistemas es una asignatura del segundo curso (primer cuatrimestre) del grado en Ingeniería de Tecnologías y Servicios de Telecomunicación de formación básica de la materia básica de Telecomunicación. Tiene asignados 4,5 créditos de teoría en el aula y 1,5 créditos de prácticas.

(c) EY-NC-ND 2016, Universitat Politècnica de València

Congreso In-Red (2016) 
En ella se presenta la teoría se señales y sistemas de tiempo continuo y discreto, necesaria para el modelado de los elementos básicos que aparecen en telecomunicaciones. Los conceptos presentados son fundamentales en el desarrollo de gran parte de las asignaturas que posteriormente se cursan en la titulación, en especial de aquellas relacionadas con el ámbito del análisis y tratamiento de señales. Desde esa perspectiva podemos considerar la asignatura como una transición entre las herramientas puramente matemáticas y su orientación hacia el ámbito específico de las telecomunicaciones.

En la asignatura se trabajan diversas competencias específicas de la materia básica de telecomunicación, siendo punto de control de dos de ellas. Así como dos competencias transversales de las que también es punto de control (se evalúa al alumno). Concretamente la CT03: Análisis y resolución de problemas trabajada en los problemas de clase y en los diversos problemas de los tres parciales que evalúan la parte teórica de la asignatura, y la CT02: Aplicación y pensamiento práctico, que nos ocupa y trabajada en dos de las cinco prácticas de la asignatura.

Por lo tanto se han aprovechado dos, de las cinco prácticas de la asignatura, para incorporar de forma integrada la formación y evaluación de la competencia transversal CT02. Los contenidos sobre los que se ha trabajado son los propios de la asignatura que por otro lado ya se evaluaban de forma habitual con ejercicios o cuestiones a resolver en la propia práctica y a entregar al final de cada una de ellas. Pero en este caso se han añadido ciertas preguntas específicas.

Concretamente, tanto en la Práctica 3 como en la Práctica 5, se ha añadido un caso práctico específico para la evaluación de la CT02. Donde se enuncia el caso con datos superfluos, para luego indicar lo que se desea que el alumno programe en MatLab.

Para la evaluación de la CT02 se han seguido los siguientes resultados de aprendizaje que se basan en la rúbrica elaborada a tal efecto de la tabla 3. Cada uno de los indicadores se han evaluado con la calificación D, C, B y A, siendo D la calificación de "no alcanzado" y A la calificación de "excelente".

Resultado de aprendizaje 1 (RA1): Identificación de los objetivos concretos siguiendo las instrucciones del ejercicio. Siendo una D si no identifica los objetivos y una A si los identifica correctamente.

Resultado de aprendizaje 2 (RA2): Evaluación de la calidad de la información proporcionada para su aplicación. Siendo una D si no distingue la información relevante y una A si justifica la selección de la información.

Resultado de Aprendizaje 3 (RA3): Propone soluciones y/o acciones concretas tras el análisis de la situación. Siendo una D si no propone soluciones concretas y una A si argumenta la solución adoptada y es correcta.

Fijando la nota final de la CT02 con el siguiente criterio:

Se ha valorado a cada alumno por separado, utilizando las 6 valoraciones disponibles de la CT02 con notas de evaluación. Tres de la Práctica 3 y otras 3 de la Práctica 5.

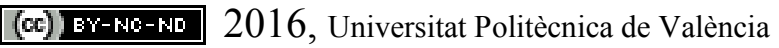


De entre los tres resultados de aprendizaje se ha valorado con un mayor peso el RA3 respecto a los otros dos, al entender que la proposición de soluciones es de un nivel mayor que la identificación de objetivos o la evaluación de la calidad.

De entre las prácticas, se ha valorado con más peso la Práctica 5 respecto a la Práctica 3 , ya que se entiende que la Práctica 3 era una primera aproximación más formativa, y la Práctica 5 ya se ha realizado una segunda evaluación de la competencia.

Es decir, se ha tenido en cuenta básicamente la valoración del RA3 de la Práctica 5, y se ha subido o bajado un nivel en función de la evolución de ese mismo RA3, de la Práctica 3 a la Práctica 5, y si hay dudas se han considerado el resto de RA de ambas prácticas (con un mayor peso de la Práctica 5). En el caso de que no hubiese valoraciones de alguna de las prácticas, se ha considerado el RA3 a la baja.

Tabla 3. Rúbrica sobre la Competencia transversal Aplicación y pensamiento práctico

\begin{tabular}{|c|c|c|c|c|c|}
\hline \multirow[b]{2}{*}{ Indicadores } & \multicolumn{4}{|c|}{ Escala de valoración } & \multirow{2}{*}{$\begin{array}{c}\text { EJEMPLOS DE } \\
\text { POSIBLES } \\
\text { EVIDENCIAS }\end{array}$} \\
\hline & No alcanzado & En desarrollo & $\begin{array}{c}\text { Bien/ } \\
\text { adecuado }\end{array}$ & $\begin{array}{l}\text { Excelente/ } \\
\text { ejemplar }\end{array}$ & \\
\hline $\begin{array}{l}\text { Identifica los } \\
\text { objetivos } \\
\text { concretos } \\
\text { siguiendo las } \\
\text { instrucciones }\end{array}$ & $\begin{array}{l}\text { No identifica } \\
\text { los objetivos }\end{array}$ & $\begin{array}{l}\text { Identifica algunos } \\
\text { objetivos pero no } \\
\text { detecta los } \\
\text { posibles } \\
\text { obstáculos para } \\
\text { su consecución }\end{array}$ & $\begin{array}{l}\text { Identifica } \\
\text { todos los } \\
\text { objetivos y } \\
\text { detecta } \\
\text { algunos } \\
\text { obstáculos } \\
\text { para su } \\
\text { consecución }\end{array}$ & $\begin{array}{l}\text { Identifica tanto } \\
\text { todos los } \\
\text { objetivos como } \\
\text { los obstáculos } \\
\text { siguiendo } \\
\text { instrucciones }\end{array}$ & $\begin{array}{c}\text { Enumera, enuncia } \\
\text { los objetivos. } \\
\text { Señala los } \\
\text { obstáculos. }\end{array}$ \\
\hline $\begin{array}{c}\text { Evalúa la } \\
\text { calidad de la } \\
\text { información } \\
\text { proporcionada } \\
\text { para su } \\
\text { aplicación }\end{array}$ & $\begin{array}{l}\text { No distingue } \\
\text { la información } \\
\text { básica y/o } \\
\text { relevante que } \\
\text { le } \\
\text { proporcionan }\end{array}$ & $\begin{array}{c}\text { Discrimina } \\
\text { parcialmente la } \\
\text { información } \\
\text { básica de la } \\
\text { irrelevante }\end{array}$ & $\begin{array}{c}\text { Discrimina } \\
\text { toda la } \\
\text { información } \\
\text { relevante que } \\
\text { le } \\
\text { proporcionan } \\
\text { después de } \\
\text { contrastar su } \\
\text { utilidad. }\end{array}$ & $\begin{array}{c}\text { Justifica la } \\
\text { selección de la } \\
\text { información } \\
\text { atendiendo a } \\
\text { aspectos como } \\
\text { veraz, } \\
\text { adecuada a la } \\
\text { situación } \\
\text { propuesta, } \\
\text { actualizada, } \\
\text { fiable, } \\
\text { accesible, etc. }\end{array}$ & $\begin{array}{c}\text { Considera } \\
\text { relevante la } \\
\text { información que } \\
\text { facilita el } \\
\text { experto/profesor, } \\
\text { reconoce, } \\
\text { selecciona y } \\
\text { analiza la } \\
\text { información } \\
\text { relevante. Estima } \\
\text { la información } \\
\text { necesaria para } \\
\text { salvar la } \\
\text { incertidumbre. }\end{array}$ \\
\hline $\begin{array}{c}\text { Propone } \\
\text { soluciones y/o } \\
\text { acciones } \\
\text { concretas tras } \\
\text { el análisis de } \\
\text { la situación }\end{array}$ & $\begin{array}{c}\text { No propone } \\
\text { soluciones y/o } \\
\text { acciones } \\
\text { concretas (se } \\
\text { pierde en } \\
\text { divagaciones, } \\
\text { ambigüedades, } \\
\text { etc.) }\end{array}$ & $\begin{array}{l}\text { Propone alguna } \\
\text { solución y/o } \\
\text { acción concreta, } \\
\text { aunque errónea } \\
\text { (no se ajusta a los } \\
\text { objetivos) }\end{array}$ & $\begin{array}{c}\text { Propone } \\
\text { alguna } \\
\text { solución y/o } \\
\text { acción } \\
\text { concreta } \\
\text { adecuada a } \\
\text { los objetivos }\end{array}$ & $\begin{array}{c}\text { Argumenta } \\
\text { coherentemente } \\
\text { las soluciones } \\
\text { y/o acciones } \\
\text { adoptadas }\end{array}$ & $\begin{array}{l}\text { Argumenta la } \\
\text { solución o la } \\
\text { acción adoptada. }\end{array}$ \\
\hline
\end{tabular}

(c) EY-NC-ND 2016, Universitat Politècnica de València

Congreso In-Red (2016) 


\subsection{Competencia CT12: Planificación y gestión del tiempo (Matemáticas II)}

La competencia transversal CT12: Planificación y gestión del tiempo se trabajó, pero no se evaluó oficialmente en las actas de Matemáticas II. Esta asignatura que se imparte en el primer cuatrimestre del segundo curso del Grado en Ingeniería Mecánica de la Escuela Técnica Superior de Ingeniería del Diseño (ETSID) de la UPV y tiene asignados en el plan de estudios 4 créditos de aula y 2 créditos de prácticas de laboratorio. Los 191 alumnos matriculados en ella durante el curso 2015-16 están distribuidos en 3 grupos, aunque las actividades que se detallan a continuación sólo se realizaron en el grupo de la tarde (39 alumnos).

Esta competencia se ha trabajado mediante ejercicios, realizados en el aula, que los alumnos entregaban al finalizar cada tema. Estos ejercicios nos sirven para valorar competencias específicas de la asignatura como: "Resolver problemas matemáticos que puedan plantearse en la ingeniería" y/o "Aprendizaje permanente: aprender nuevos métodos y teorías que se adapten a nuevas situaciones", pero a su vez pueden ser utilizados para evaluar esta competencia transversal considerando otras herramientas como las listas de control.

En estas listas el profesor registra evidencias relacionadas con la adquisición de esta competencia como por ejemplo si los alumnos:

- Traen el material necesario para realizar la actividad

- Tienen el material que necesitan organizado

- En caso de duda, buscan alternativas en la resolución de un ejercicio, preguntando a un compañero o bien al profesor

- Entregan la tarea a tiempo

De manera que además de la rúbrica facilitada por la UPV para esta competencia, las lista de control elaboradas por el profesor han resultado muy útiles para asignar a cada alumno el nivel de dominio de esta competencia en nuestra asignatura que, aunque no ha figurado en el acta, si ha sido evaluada a modo de prueba.

\subsection{Competencia CT09: Pensamiento crítico (Sistemas Electrónicos)}

La competencia transversal CT09: Pensamiento crítico se trabajó, pero no se evaluó oficialmente en las actas de Sistemas Electrónicos (SSEE). Esta asignatura que se imparte en el primer cuatrimestre del tercer curso del Grado en Ingeniería en Tecnologías Industriales (ETSII) de la UPV. Esta materia tiene asignados en el plan de estudios 3.6 créditos de aula y 0.9 créditos de prácticas de laboratorio. Los 319 alumnos matriculados en ella durante el curso 2015-16 están distribuidos en 6 grupos.

La competencia Pensamiento crítico se ha evaluado mediante una pregunta en cada una de las tres sesiones de prácticas de la asignatura.

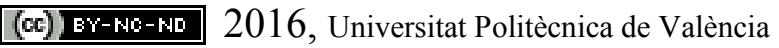

Congreso IN-RED (2016) 
En cada una de estas prácticas el alumno debe realizar un trabajo previo que consiste en el diseño de ciertos circuitos electrónicos. El alumno debe calcular y seleccionar los valores de determinados componentes de los circuitos para que se cumplan las especificaciones impuestas. En el desarrollo de la práctica, el alumno comprueba experimentalmente el funcionamiento de los circuitos, contrasta los resultados y observaciones obtenidas en el laboratorio con sus conocimientos y con el trabajo previo realizado y valora la coherencia de los resultados.

$\mathrm{Al}$ terminar la práctica, cada grupo de alumnos entrega la memoria con el trabajo previo, las mediciones realizadas en el laboratorio y las preguntas diseñadas para valorar esta competencia (una por práctica). En estas preguntas el alumno debe contrastar los resultados y observaciones con sus conocimientos y valorar la coherencia de los resultados.

La nota de la competencia ce calcula con el número de preguntas contestadas correctamente:
A (Excelente): 3
B (Adecuado): 2
C (En desarrollo): 1
D (No alcanza): 0

\subsection{Competencia CT10 Conocimiento de problemas contemporáneos (Tratamiento biológico de aguas residuales)}

Tratamiento biológico de aguas residuales es una asignatura optativa del Máster en Ingeniería Química de 6 ECTS y el número de alumnos durante el presente curso académico ha sido de 7 .

La metodología empleada para trabajar esta competencia ha sido de forma colaborativa en el aula mediante debate. El tema del debate constituye uno de los grandes problemas contemporáneos de la depuración de aguas "la gestión final del fango". El fango es uno de los residuos finales del proceso de depuración, por lo que a medida que se han construido las depuradoras de aguas residuales se han ido generando muchas más toneladas de fango. Para que los alumnos fueran bien preparados al debate, se les pidió que vinieran preparados habiendo consultado: legislación vigente sobre uso del fango en la agricultura (la principal forma de gestión en España), alternativas de gestión e informes de la Unión Europea

En la clase donde se planteó el debate se comenzó viendo el video "sludge diet" donde se denuncia la relación entre el abonado con fango de depuradora y enfermedades respiratorias surgidas en una pequeña población de Estados Unidos. Este video se pudo ver en TVE en el programa Documentos TV, creando una polémica adicional ya que "sludge" fue traducido por compost, lo que también fue discutido en el debate.

Para llevar a cabo la evaluación, los alumnos, tras el debate, tuvieron que realizar un informe individual en el que respondían unas cuestiones planteadas, que incluían una

(cc) EY-NC-ND 2016, Universitat Politècnica de València

Congreso In-Red (2016) 
propuesta razonada de cuál es la forma que ellos elegirían de gestionar el fango. Por tanto, realmente se estaba también trabajando las competencias de CT09: Pensamiento crítico y la CT08: Comunicación efectiva.

Para la evaluación se empleó la rúbrica propuesta por la UPV, eso sí, adaptada al debate planteado. Concretamente se empleó la de dominio de nivel II, pese a ser alumnos de máster (a los que le correspondería un dominio de nivel III). Ello es debido a que al ser el primer curso donde se trabajan estas competencias, los estudiantes no habían trabajado ésta con anterioridad.

El resultado fue muy bueno pues los 7 alumnos fueron evaluados con el máximo nivel de adquisición (A, excelente), lo que nos lleva a pensar si quizás se hubiera podido emplear la rúbrica de nivel III.

La competencia CT06: Trabajo en equipo y liderazgo también se ha evaluado en la asignatura Tratamiento biológico de aguas residuales. Cabe decir que en esta signatura, las actividades que se hacen en grupo ( 2 casos prácticos y prácticas de laboratorio) contribuyen al $60 \%$ de la nota final, mientras que el examen es el $40 \%$ de la nota final.

La CT06 se ha evaluado en las prácticas de laboratorio en sí y en la redacción de los informes correspondientes. Estas prácticas se han llevado a cabo en dos grupos (uno de 4 y otro de 3 estudiantes) y consistían en la operación de un reactor de laboratorio de tratamiento de aguas residuales (en las dos primeras prácticas se trabajaba con aguas residuales urbanas y en las dos últimas con aguas residuales industriales).

La evaluación durante la práctica se ha realizado mediante observación empleando la rúbrica de la UPV de nivel de dominio III, mientras que para los informes presentados se ha empleado una rúbrica de elaboración propia donde se recogían más aspectos de la competencia CT08: Comunicación efectiva que de la CT09: Trabajo en grupo.

Cabe destacar los siguientes aspectos a modo de resumen:

- Con solo 7 alumnos en laboratorio (2 grupos), la rúbrica del ICE se puede aplicar perfectamente pues el profesor es capaz de observar todo lo que sucede en todos los grupos: distribución de tareas, quien contribuye al compromiso colectivo del equipo, quien es líder, etc.

- Se ha empleado la rúbrica de nivel de dominio III porque los alumnos han trabajado esa competencia durante el grado en ingeniería química, ya que han tenido asignaturas en donde las prácticas son un $100 \%$ de la metodología docente.

- $\quad$ El resultado ha sido excelente, lo cual no es sorprendente dada la formación previa que estos estudiantes han tenido en el laboratorio de ingeniería química.

\section{Análisis de las competencias transversales}

En este apartado se muestra el análisis realizado sobre las competencias transversales que se han evaluado en las asignaturas anteriormente introducidas.

(c)) EY-NC-ND 2016, Universitat Politècnica de València

Congreso IN-RED (2016) 
En la Figura 2 se muestran los resultados tras evaluar la competencia transversal CT10 Conocimiento de problemas contemporáneos en la asignatura Matemática Discreta. En ella se puede apreciar una buena correlación entre las notas finales y las de la competencia que analizamos. Sin embargo observamos que hay valores en que ambas puntuaciones no cuadran, como consecuencia podemos inferir de que la proporción entre el peso de las notas del examen de materia de aula y las de prácticas es de 3 a 1.

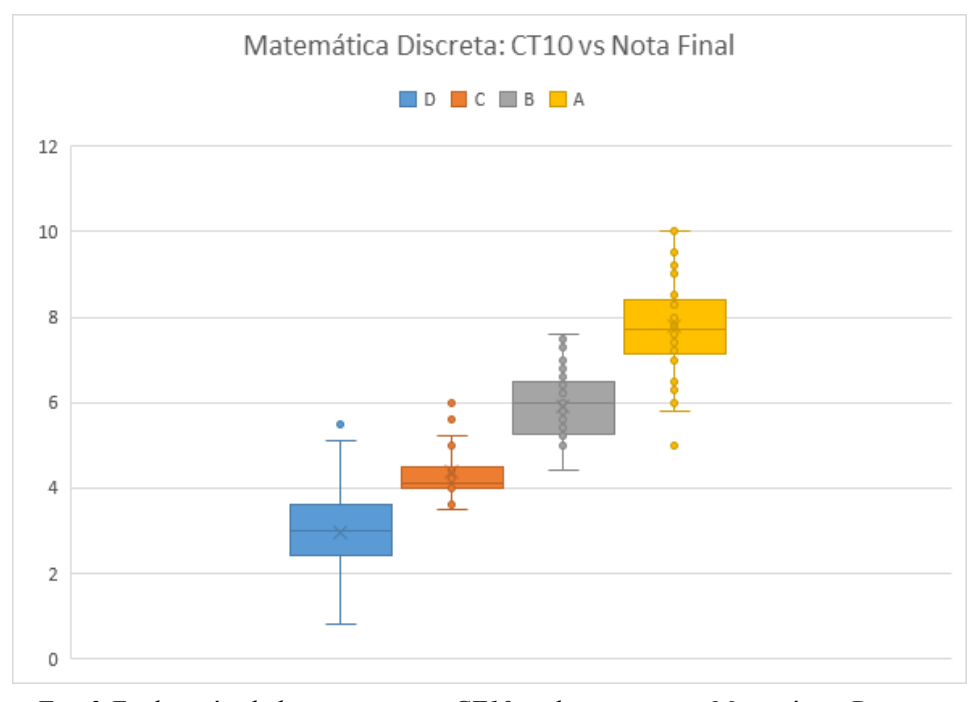

Fig. 2 Evaluación de la competencia CT10 en la asignatura Matemática Discreta

En la Figura 3 se muestran los resultados tras evaluar la competencia transversal CT02: Aplicación y pensamiento práctico en la asignatura Señales y Sistemas.

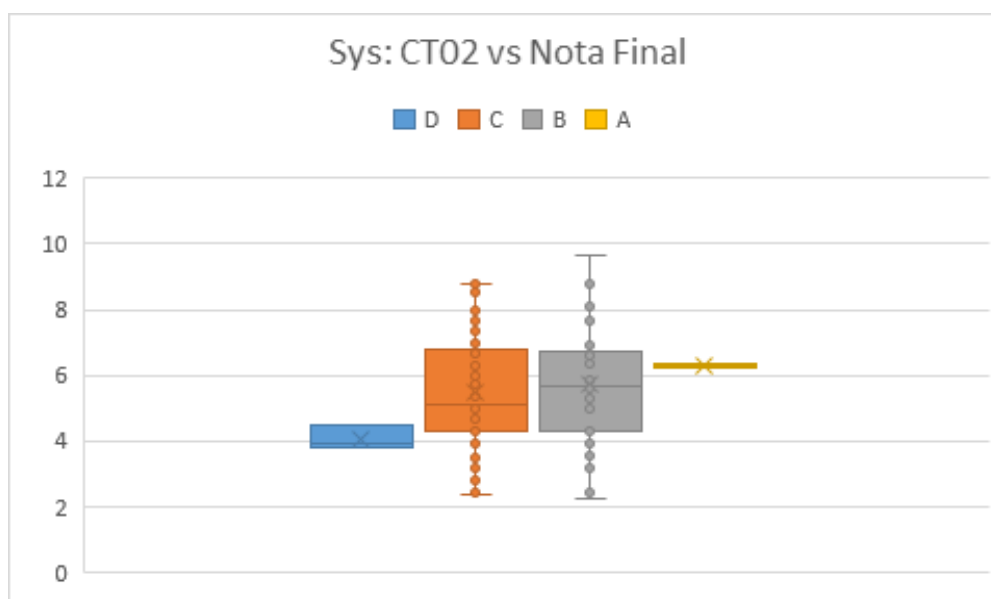

Fig. 3 Evaluación de la competencia CT02 en la asignatura Señales y Sistemas 
En este caso se puede apreciar como el hecho de utilizar unos ejercicios específicos e independientes de la nota de la práctica ha provocado que no exista una clara correlación entre la nota final de la asignatura y la evaluación de la CT02. Incluso se dan casos de alumnos que tienen notas de $\mathrm{C}$ e incluso de $\mathrm{B}$ en la competencia y sin embargo no logran superar la asignatura. Hay que destacar en este caso, que la nota final de la asignatura se corresponde en su mayor parte por la nota de problemas de los exámenes (80\%) siendo la nota de prácticas de solo un $20 \%$, lo que puede explicar en parte esta tendencia. Sin embargo si se observa la Figura 4, se puede ver como tampoco se correla directamente con la propia nota de las prácticas, ya que efectivamente se están observando cuestiones diferentes.

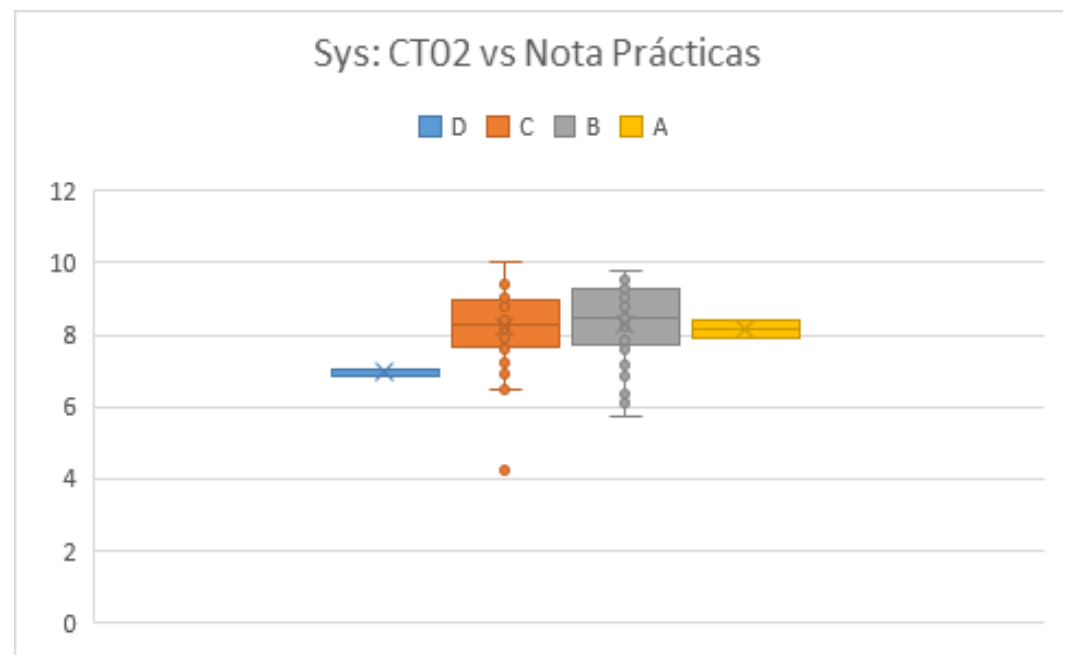

Fig. 4 Comparativa de la evaluación de CT02 en función de la nota de prácticas en Señales y Sistemas

En la Figura 5 se muestran los resultados tras evaluar la competencia transversal CT12: Planificación y gestión del tiempo en Matemáticas II. Observamos que destacar con nota A (excelente) en esta competencia tiene muy diversos desempeños en la asignatura de Matemática II. Por otro lado cabe destacar que alumnos que han superado la asignatura con notas similares no han demostrado el mismo nivel de dominio de esta competencia lo que, desde nuestro punto de vista resulta totalmente coherente puesto que esta competencia transversal no tiene una especial relación con las competencias específicas evaluadas en esta materia. 


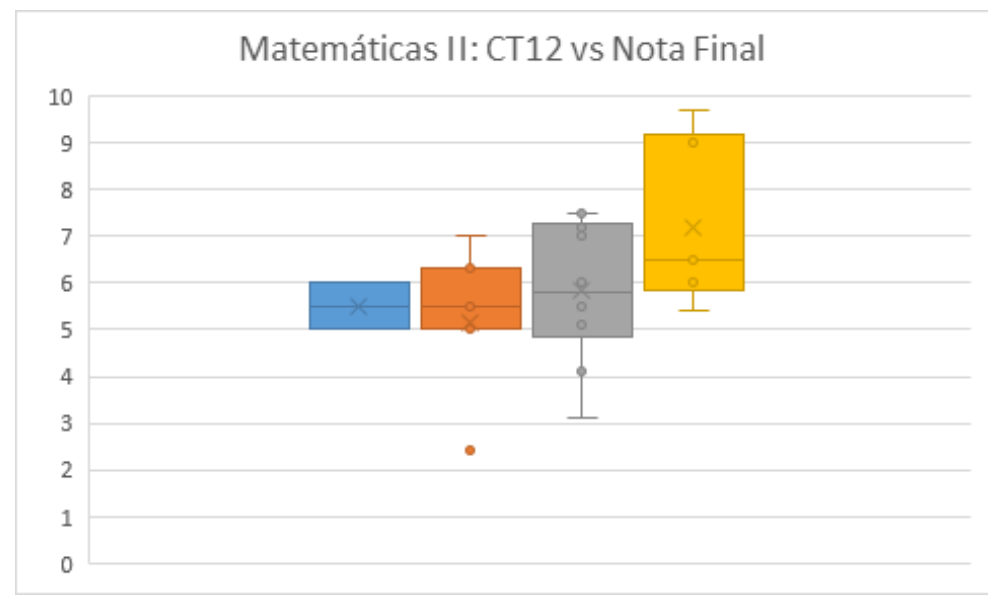

Fig. 5 Evaluación de la competencia CT12 en la asignatura Matemáticas II

En la Figura 6 se muestran los resultados tras evaluar la competencia transversal CT09: Pensamiento crítico en la asignatura Sistemas Electrónicos. En esta figura se puede observar que no existe una correlación directa entre la calificación de la competencia transversal evaluada y la nota final del alumno, aunque el porcentaje de alumnos que obtienen la peor calificación en la competencia transversal es mayor entre los alumnos que no superan la asignatura. La ausencia de correlación directa se puede deber a que la evaluación de esta competencia se ha llevado a cabo mediante unas cuestiones específicas que no se han utilizado para la nota final de la asignatura.

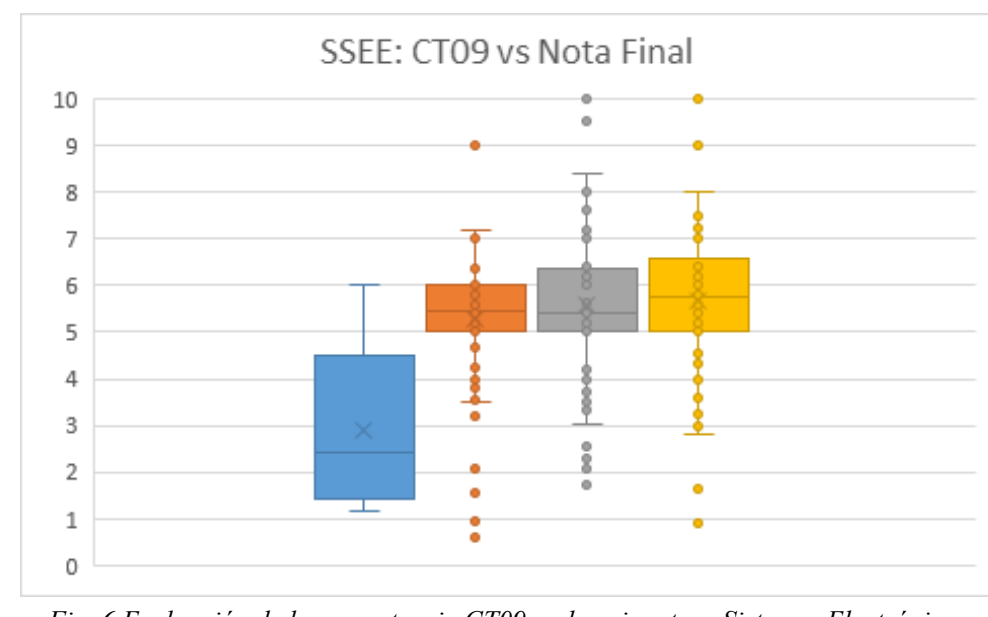

Fig. 6 Evaluación de la competencia CT09 en la asignatura Sistemas Electrónicos

En la asignatura Tratamiento Biológico de Aguas residuales los 7 alumnos han obtenido la calificación de sobresaliente, por lo que no se incluye un gráfico relacionando la evaluación de la competencia CT10 con la nota final de la asignatura. 


\section{Conclusiones}

En este artículo se muestra la metodología utilizada para trabajar con diversas competencias transversales en diferentes asignaturas pertenecientes a diversas titulaciones de ingeniería de la UPV. Todas estas asignaturas son impartidas por profesores del grupo de innovación docente EITACURTE, los cuales realizan reuniones periódicas para intercambiar ideas en sus experiencias docentes.

En este caso se han analizado las competencias CT02: Aplicación y pensamiento práctico, CT09: Pensamiento crítico, CT10: Conocimiento de problemas contemporáneos y CT12: Planificación y gestión de tiempo y en todas ellas se han obtenido resultados satisfactorios.

En el caso de la CT02 en Señales y Sistemas, al haber sido evaluada con un ejercicio específico y diferenciado del resto de ejercicios que componían la nota de prácticas (dos de las cinco prácticas) se observa que no hay una correlación directa entre las calificaciones de la competencia transversal y la nota de las prácticas, ni entre la nota de la competencia y la nota final. Algo similar ocurre con la CT09 en Sistemas Electrónicos y con la CT12 en Matemáticas II, donde al introducirse además de los ejercicios propios de la asignatura, otros elementos de evaluación, como ejercicios específicos para la competencias o listas de control del profesor, existe un abanico muy amplio de relaciones entre la nota de la competencia y la nota final de la asignatura.

Sin embargo, las competencias donde la nota se ha obtenido directamente de alguna parte de la asignatura, como por ejemplo en el caso de la competencia CT10 en Matemática Discreta, en general la correlación entre la nota de la competencia y de la propia asignatura es mucho más directa.

En futuros trabajos se pretende verificar si ocurren cosas similares a las encontradas en la asignatura Señales y Sistemas en otras asignaturas. Además, se pretende mejorar la metodología empleada a partir de los análisis realizados.

\section{Referencias}

ABET. (1997). Engineering criteria 2000: Criteria for accrediting programs in engineering in the United States. Baltimore, MD: Author.

ABET Engineering Accreditation Commission. (2008). Criteria for accrediting engineering programs: Effective for evaluations during the 2009-2010 accreditation cycle. Retrieved from http://www.abet.org/Linked\%20Documents-UPDATE/Criteria\%20and\%20PP/E001\%2009$10 \% 20$ EAC $\% 20$ Criteria $\% 2012-01-08$.pdf

BJORKLUND, S.A.; PARENTE, J.M.; SATHIANATHAN, D. ; (2004) "Effects of Faculty Interaction and Feedback on Gains in Student Skills" Journal of Engineering Education. Vol. 93, Issue 2, p 153-160

GONZÁLEZ, J.,WAGENAAR, R. (2003). Tuning Educational Structures in Europe. Informe Final. Bilbao: Universidad de Deusto

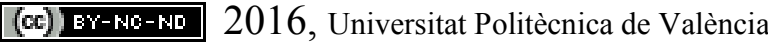

Congreso IN-RED (2016) 
Silvia Álvarez Blanco, Ignacio Bosch Roig, Cristina Jordán Lluch, Jaime Lloret, José Antonio Mendoza, Lucía Romero Pérez, Esther Sanabria-Codesal y Maria Cinta Vincent-Vela

JAMIESON, L. 2007. Experiencing engineering. Main Plenary. ASEE Annual Conference, Honolulu, Hawaii, http://www.asee.org/conferences/ anual/2007/Highlights.cfm\#main

KILGORE, D., ATMAN, C. J., YASUHARA, K., BARKER, T. J., \& MOROZOV, A. (2007). Considering context: A study of first-year engineering students. Journal of Engineering Education, 96(4), 321-334.

KYOUNG RO, H., MERSON, D., B LATTUCA, L.R., TERENZINIB, P. (2015). “ Validity of the Contextual Competence Scale for Engineering Students" en Journal of Engineering Education. Jan2015, Vol. 104, Issue 1, p35-54.

LLORET, J., JIMÉNEZ, J. M., BORONAT, F., TOMÁS, J., DÍAZ, J.R., Utilización de diversas metodologías didácticas para desarrollar las habilidades de los estudiantes de Ingeniería Técnica de Telecomunicaciones, Congreso Internacional de Docencia Universitaria e Innovación (CIDUI 2006), Barcelona, España, Barcelona 5 - 7 de Julio 2006.

REDISH. E.F. ; SMITH, K.A. (2008) “ Looking Beyond Content: Skill Development for Engineers “ Journal of Engineering Education. Vol. 97, Issue 3, p295-307

SANABRIA-CODESAL, E., BOSCH, I., VINCENT-VELA, M.-C., LLORET, J., ÁLVAREZBLANCO, S., ROMERO PÉREZ, L., Análisis de las Dimensiones Competenciales Incluidas en Diferentes Asignaturas en Ingenierías, Jornadas de Innovación Educativa y Docencia en Red (In-Red 2014), Valencia (España), 15-16 de Julio 2014

SENDRA, S., CANOVAS, A., GARCÍA, M., LLORET, J., Método de evaluación cooperativa en clases prácticas de redes de ordenadores, EAA-Jornadas de Innovación, Julio 8-10, 2009. Valencia, España.

(cc) EY-NC-ND 2016, Universitat Politècnica de València

Congreso In-Red (2016) 